\title{
Extracellular Matrix of Cartilage and Meniscus by ESEM and Immunogold Labeling
}

\author{
Monica Nelea*, Anik Chevrier*, Anne Gigout*, Adele Changoor*, Michael D. Buschmann* \\ *Department of Chemical Engineering, Institute of Biomedical Engineering, Ecole Polytechnique of Montreal, Qc, \\ Canada $\mathrm{H} 3 \mathrm{C} 3 \mathrm{~A} 7$
}

Introduction. Articular cartilage and meniscus are biological tissues with a pivotal biomechanical function in knee and which frequently undergo injury and degeneration. Unveiling the structure of cartilage and meniscus and the organization of extracellular matrix that comprises these tissues at micro-and nanometric scales is of important in assessing novel treatments and therapies for their repair and regeneration. The primary extracellular component of articular cartilage is collagen type II, which forms a highly organized fibrillar network with a fundamental role in the biomechanical properties of this tissue. Menisci play a central role in the knee joint, protecting the articular cartilage from excessive stress, absorbing mechanical shocks, and stabilizing the joint [1]. In menisci, collagen type I is the primary constituent with minor amounts of collagen type II, III, V and VI. Cell-matrix and cell-cell interactions in cartilage are of crucial importance in mediating cartilage development, homeostasis, degradation, and cell survival [2]. We report an investigation of extracellular matrix structure and organization of cartilage and meniscus tissues of different origin by environmental scanning electron microscopy (ESEM) imaging and immunogold labeling ESEM imaging. One goal of this research study is to qualitatively and quantitatively assess collagen organization in cartilage and meniscus sections and to localise different types of collagen in cartilage or meniscus. Another objective is to outline the importance of ESEM as high-resolution imagery as tool to localize chondrocyte-specific proteins involved in cartilage development by immunogold labelling and assess their distribution.

Methods. Samples included full-thickness cartilage disks $(n=4)$ from the trochlea of an adult equine knee (stifle) joint, repair and non-operated osteochondral tissues collected from a human knee $(\mathrm{n}=5)$ that had undergone a bone marrow stimulation cartilage repair procedure on the medial femoral condyle (MFC), and menisci from skeletally mature New Zealand white rabbits $(n=2)$, Suffolk-Dorset sheep $(n=2)$ and humans $(n=2)$. For studies with cells, chondrocyte aggregates formed after $18 \mathrm{~h}$ suspension culture were fixed, blocked, probed with first primary antibodies for type II Collagen and COMP and with secondary antibodies conjugated to $20 \mathrm{~nm}$ or $10 \mathrm{~nm}$ gold particles (1/40, British Biocell International and 1/40 Aurion), and then post-fixed. We used an ESEM Quanta 200 FEG instrument (FEI Co., Hillsboro, OR). Prior to electron microscopy observations, low magnification identification of cartilage zones based on collagen orientation was performed with polarized light microscopy (PLM). Adjacent cartilage sections observed in SEM were scored for collagen orientation and collagen density on a scale of 0 to 2 . Orientation that is appropriate for the particular zone scored a 2 (e.g. vertical in the radial zone), while inappropriate orientation scored a 0 , with 1 being intermediate. Similar analysis was performed on human repair samples at various regions of interest (ROI) corresponding to sites with irregular cartilage repair tissue that could not be described by the typical zonal structure of normal articular cartilage. Collagen fibril diameter was quantified systematically by image processing (ESEM XT Docu v.3.2 software).

Results. SEM scores for collagen orientation and density were consistently 2 for all equine cartilage samples and less or equal for the osteochondral MFC repair samples. Collagen fibril diameters are substantially larger in the deep zone (140$200 \mathrm{~nm}$ ) and decrease in size towards the articular surface (40-140 nm) (Fig 1 and Fig 2). The application of scoring system helped to distinguish repair cartilage from normal cartilage and to classic repair cartilage of different degrees, giving an assessment of repair efficacy. For menisci, ESEM revealed the complex arrangement of meniscal collagen in three distinct layers (Fig 3): (a) a fibril network covering the femoral and tibial surfaces, (b) a lamellar layer oriented towards the femoral and tibial surface and finally (c) a central main portion composed of circumferentially arranged fibers with occasional radial tie fibers [3]. ESEM unveiled the ultrastructure of chondrocyte aggregates formed in suspension culture (Fig. 4). These analyses showed that chondrocytes in aggregates taken after 18 hours of suspension culture were not in direct contact one to other, but linked by a randomly-oriented, dense fibril network (Fig. 4A), with extensive cross-linking and fibril bonding (Fig. 4B). Fibrils appeared to sprout out of the cells; some small fibrils could merge to produce larger structures growing in diameter (Fig. 4B). In some locations fibrils were wider, creating a loose fibre network (Fig. 4B, D), while other locations displayed a denser network with smaller diameter structures (Fig. 4C). Fibril diameter varied from 20 to $180 \mathrm{~nm}$, as measured on ESEM images, since gold coating for high vacuum observations significantly increased fibril width. Immunogold labeling revealed that the intercellular fibre network was positive for collagen type II and COMP confirming the presence of these molecules spanning the intercellular space in chondrocytes aggregates formed after 18h (Fig. 4C, D), and affirming their possible involvement in aggregate formation and organisation [4]. 
Conclusion. We demonstrated the capability of ESEM as an imaging tool for the characterization of articular cartilage and meniscus and the extracellular matrix that makes up these tissues at micro-, submicro- and nano-scales. The systematic scoring of collagen orientation was proven efficacious to categorize the quality of repair tissues, and a useful method to assess performance of treatment of disfunctional cartilage. The ESEM was capable of visualizing and distinguishing minute details of samples like the fine collagen fibers of nanometer diameter or gold nanoparticles conjugated on molecular probes (antibodies) is of great importance in deciphering structural organization of collagen and assembly of cell matrix in cartilage and meniscus will high impact in the developing of novel models for cartilage research for humans.

\section{References}

[1] Walker PS, Erkman MJ. The role of the menisci in force transmission across the knee. Clin Orthop Relat Res. 1975; 109: 184-92.

[2] Woods, A., G. Wang, and F. Beier. 2007. Regulation of chondrocyte differentiation by the actin cytoskeleton and adhesive interactions. J Cell Physiol. 213:1-8.

[3] Chevrier A, Nelea M, Sun J, Hurtig MB, Hoemann CD and Buschmann MD. Meniscus Structure in Human, Sheep and Rabbit for Animal Models of Meniscus Repair. Journal of Orthopedic Research, in press.

[4] Gigout A, Jolicoeur M, Nelea M, et al. 2008. Chondrocyte Aggregation in Suspension Culture Is GFOGER-GPPand beta 1 Integrin-dependent 283:31522-31530, Journal of Biological Chemistry.

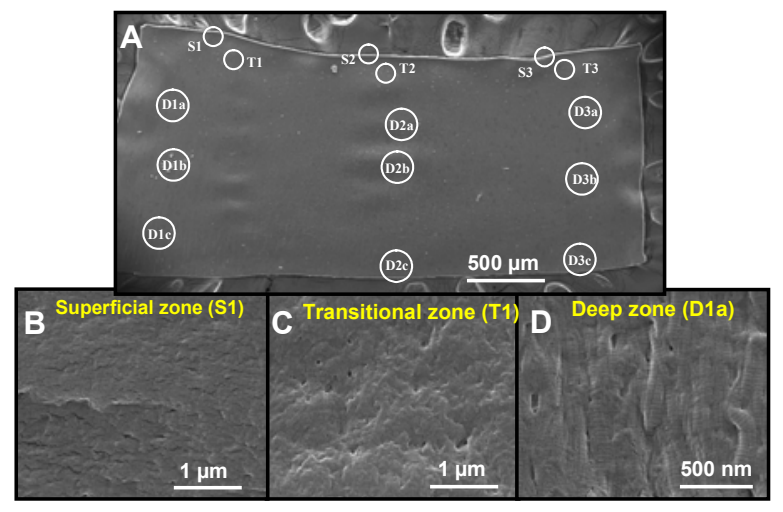

Figure 1: Low magnification ESEM (high vacuum mode) image showing locations for high magnification imaging (A). High magnification $(80,000 \times)$ images showing collagen orientation and density in each zone (B, C and D).

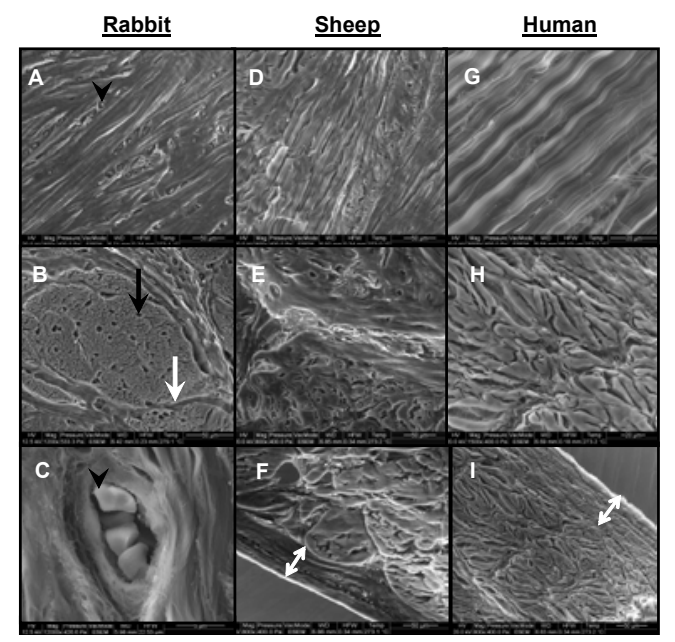

Figure 3: ESEM pictures of menisci from rabbit (A, B, C), sheep (D, E, F) and human (G, H, I) sectioned in the coronal (A, C, D. G) and horizontal (B, E, F, H, I) orientation.

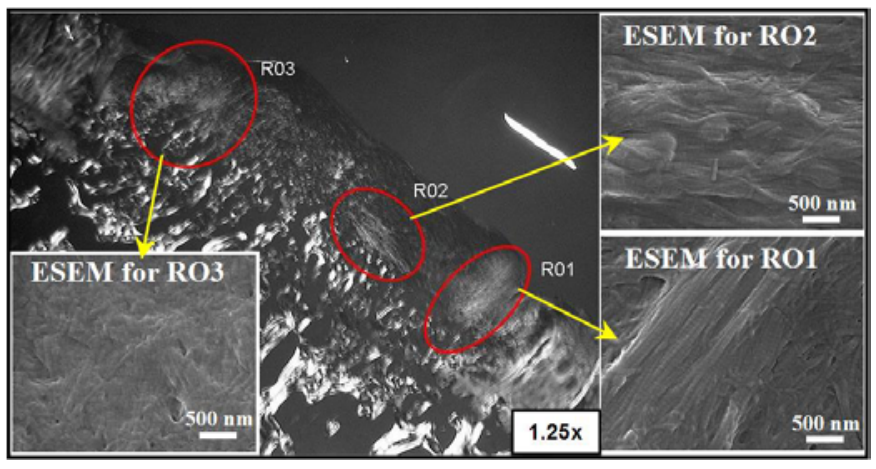

Figure 2: Repair medial femoral condyle cartilage showing regions of birefringent tissue emanating from microfracture holes (ROI-1 \& ROI-3) and oriented tissue between (ROI-2) with corresponding high magnification SEM images.
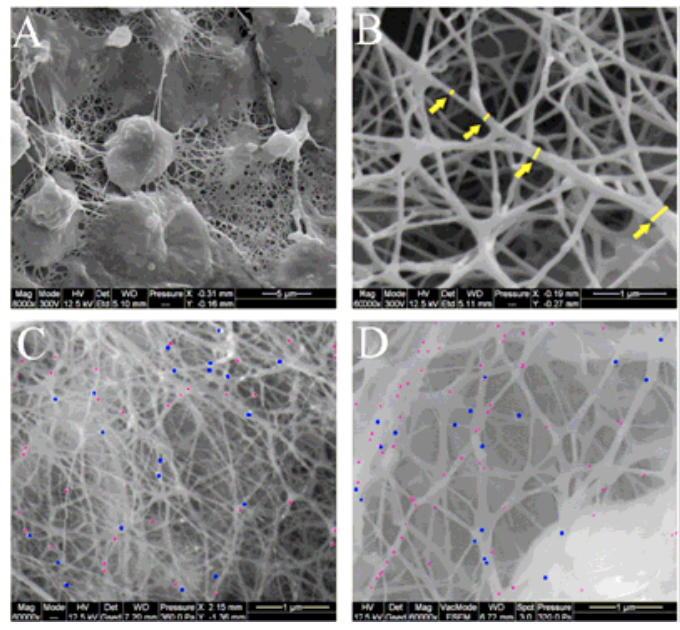

Figure 4: Chondrocyte aggregates after $18 \mathrm{~h}$ of culture in suspension observed in ESEM in high vacuum mode (A-B) and in environmental mode (C-D). 